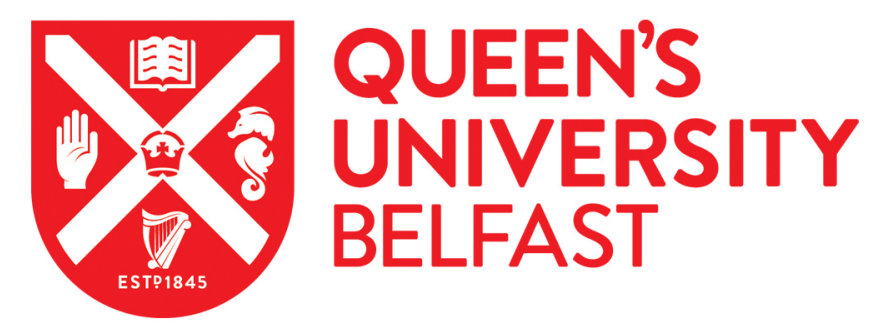

\title{
High flux, beamed neutron sources employing deuteron-rich ion beams from $\mathrm{D} 2 \mathrm{O}$-ice layered targets
}

Alejo, A., Krygier, A. G., Ahmed, H., Morrison, J. T., Clarke, R. J., Fuchs, J., Green, J. S., Green, J. S., Jung, D., Kleinschmidt, A., Najmudin, Z., Nakamura, H., Norreys, P., Notley, M., Oliver, M., Roth, M., Vassura, L., Zepf, M., Borghesi, M., ... Kar, S. (2017). High flux, beamed neutron sources employing deuteron-rich ion beams from D 2 O-ice layered targets. Plasma Physics and Controlled Fusion, 59(6), 064004. http://stacks.iop.org/0741$3335 / 59 / i=6 / a=064004$

\section{Published in:}

Plasma Physics and Controlled Fusion

Document Version:

Peer reviewed version

Queen's University Belfast - Research Portal:

Link to publication record in Queen's University Belfast Research Portal

Publisher rights

(C) 2017 IOP Publishing Ltd. This work is made available online in accordance with the publisher's policies. Please refer to any applicable terms of use of the publisher.

\section{General rights}

Copyright for the publications made accessible via the Queen's University Belfast Research Portal is retained by the author(s) and / or other copyright owners and it is a condition of accessing these publications that users recognise and abide by the legal requirements associated with these rights.

Take down policy

The Research Portal is Queen's institutional repository that provides access to Queen's research output. Every effort has been made to ensure that content in the Research Portal does not infringe any person's rights, or applicable UK laws. If you discover content in the Research Portal that you believe breaches copyright or violates any law, please contact openaccess@qub.ac.uk. 


\title{
High flux, beamed neutron sources employing deuteron-rich ion beams from $\mathrm{D}_{2} \mathrm{O}$-ice layered targets
}

\author{
A. Alejo ${ }^{1}$, A.G. Krygier ${ }^{2}$, H. Ahmed ${ }^{1}$, J.T. Morrison $^{3}$, R.J. \\ Clarke $^{4}$, J. Fuchs ${ }^{5}$, A. Green ${ }^{1}$, J.S. Green ${ }^{4}$, D. Jung ${ }^{1}$, A. \\ Kleinschmidt $^{6}$, Z. Najmudin ${ }^{7}$, H. Nakamura ${ }^{7}$, P. Norreys ${ }^{1}$, \\ M. Notley ${ }^{4}$, M. Oliver ${ }^{4}$, M. Roth ${ }^{6}$, L. Vassura ${ }^{5}$, M. Zepf ${ }^{1}$, \\ M. Borghesi ${ }^{1}$, R.R. Freeman ${ }^{2}$, S. Kar ${ }^{1, *}$ \\ 1 Centre for Plasma Physics, School of Mathematics and Physics, Queen's \\ University Belfast, Belfast BT7 1NN, United Kingdom \\ 2 Department of Physics, The Ohio State University, Columbus, Ohio 43210 , \\ USA \\ 3 Propulsion Systems Directorate, Air Force Research Lab, Wright Patterson \\ Air Force Base, Ohio 45433, USA \\ ${ }^{4}$ Central Laser Facility, Rutherford Appleton Laboratory, Didctor, Oxfordshire \\ OX11 0QX, UK \\ 5 LULI - CNRS, Ecole Polytechnique, CEA : Université Paris-Saclay ; UPMC \\ Univ Paris 06 : Sorbonne Universites - F-91128 Palaiseau Cedex, France \\ 6 Institut für Kernphysik, Technische Universität Darmstadt, Schloßgartenstraße \\ 9, Darmstadt D-64289, Germany, \\ 7 Blackett Laboratory, Department of Physics, Imperial College, London SW7 \\ $2 \mathrm{AZ}, \mathrm{UK}$ \\ E-mail: s.kar@qub.ac.uk
}

\begin{abstract}
A forwardly-peaked bright neutron source was produced using a laser-driven, deuteron-rich ion beam in a pitcher-catcher scenario. A protonfree ion source was produced via target normal sheath acceleration from $\mathrm{Au}$ foils having a thin layer of $\mathrm{D}_{2} \mathrm{O}$ ice at the rear side, irradiated by sub-petawatt laser pulses $(\sim 200 \mathrm{~J}, \sim 750 \mathrm{fs})$ at peak intensity $\sim 2 \times 10^{20} \mathrm{~W} \mathrm{~cm}^{-2}$. The neutrons were preferentially produced in a beam of $\sim 70^{\circ}$ cone along the ion beam forward direction, with maximum energy up to $\sim 40 \mathrm{MeV}$ and a peak flux along the axis $\sim 2 \times 10^{9} \mathrm{n} / \mathrm{sr}$ for neutron energy above $2.5 \mathrm{MeV}$. The experimental data is in a good agreement with the simulations carried out for $d(d, n)^{3}$ He reaction using the deuteron beam produced by the ice-layered target.
\end{abstract}




\section{Introduction}

Laser-driven neutron sources have recently attracted a great deal of attention due to their broad range of potential applications, favoured by a number of intrinsic advantages transcending conventional accelerator-based spallation sources $[1,2,3,4]$, such as short pulse duration, minimal shielding requirements, reduced cost and compactness. Among various laser based schemes used for neutron generation, the so called pitcher-catcher scenario [5] has been shown numerically $[6]$ and experimentally $[7,8,9,10]$ to produce highest neutron fluxes with pronounced anisotropy in neutron emission, favourable for beam transport and moderation. In this scheme light ions are accelerated from a pitcher target (the target irradiated by intense laser) and are allowed to collide with a secondary converter, where fusion reactions take place producing a directional, bright neutron source.

Light ions can be accelerated efficiently using intense short pulse lasers via a variety of established and emerging acceleration mechanisms, such as Target Normal Sheath Acceleration (TNSA) [11], radiation pressure acceleration $[12,13]$, relativistic transparency enhanced acceleration schemes $[14,15$, 16, 17], guided post-acceleration by laser-driven microcoils [18, 19]. Although TNSA is the most studied and robust acceleration mechanism, it is prone to accelerating preferentially the lightest ion species at the rear surface of the target, i.e. the protons produced from the hydrocarbon and water vapour contaminant layers present on the target surface $[20,21]$. Although the laser-driven, 10s of $\mathrm{MeV}$ protons can be efficiently deployed for neutron production $[4,22,23]$, there are several high cross-section reactions for neutron production, such as $\mathrm{d}(\mathrm{d}, \mathrm{n})^{3} \mathrm{He}$ and $\mathrm{d}\left({ }^{7} \mathrm{Li}, \mathrm{n}\right)^{8} \mathrm{Be}$ reactions, employing energetic $(\mathrm{MeV})$ deuteron beams [24, 25]. Therefore mechanisms capable of accelerating bulk ion species and their subsequent use for neutron production are currently being explored by several groups $[7,8,9,24$, $26]$.

Due to many stringent requirements in terms of laser and target parameters for the aforementioned bulk acceleration mechanisms using ultra-thin foils, several methods for producing deuteron-rich ion beams based on the TNSA mechanism have been proposed [27, 28, 29]. Protons are preferentially accelerated in the TNSA mechanism because of their highest charge to mass ratio and the unavoidable hydrocarbon contaminant layer at the target surface. Therefore efficient acceleration of heavier ion species can be achieved either by removing the proton rich contaminant layers from the target surfaces, for example by resistive heating [30, 31] and using an ion sputter gun [32], or by over coating the rear surface with a layer of hydrogen-free substance just before the interaction. The method proposed by Morrison et al. [29] is based on the deposition of a thin layer of $\mathrm{D}_{2} \mathrm{O}$ ice on the rear surface of the target foil. The ice layer buries the proton-rich contaminant layers and therefore optimises the acceleration of deuterium, being the lightest ion species at the rearmost surface of the laser-irradiated target. A 99\%-pure deuteron beam was demonstrated recently by Krygier et al. [33] using $\mathrm{D}_{2} \mathrm{O}$ ice covered targets at Vulcan petawatt laser, with a laser-to-deuteron energy conversion efficiency above $9 \%$.

Here we report on the bright, directional neutron beam generated using the deuteron-rich beam obtained during the same experimental campaign. The deuteron beam produced by the ice-layered target was characterised using several Thomson parabola spectrometers positioned along different angles with respect to the target normal. By using a secondary deuterised-plastic (CD) converter, peak neutron flux in excess of $10^{9} \mathrm{n} / \mathrm{sr}$ (for neutrons of energy above $2.5 \mathrm{MeV}$ ) in a forwardly directed beam of $\sim 70^{\circ}$ Full-Width-at-Half-Maximum was produced via the $\mathrm{d}(\mathrm{d}, \mathrm{n})^{3}$ He reaction, which is in good agreement with a simulation using the numerical modelling described in Ref. [34].

The remaining of the paper is structured as follows. In Sec. 2 the experimental setup is discussed. The spectral characterisation of the deuteron-rich ion beam, in comparison with TNSA-driven sources from typical deuterated and non-deuterated targets, is discussed in Sec. 3, followed by (in Sec. 4) the spectrally and angularly resolved characterisation of the neutron beam produced from different target configurations (no-ice pitcher, ice-layered pitcher and ice-layered pitcher with catcher). The comparison between the experimental and simulated neutron beam spatial profiles is also shown in Sec. 4.

\section{Experimental setup}

The experiment was carried out using the petawatt arm of the Vulcan laser (Central Laser Facility, UK) [35]. The laser beam was focussed down to $\sim 5 \mu \mathrm{m}$ spot on the target by using an $f / 3$ off-axis parabola, after being reflected off a plasma mirror in order to improve the contrast ratio between the main pulse and the preceding pedestal. The Vulcan laser, of central wavelength $\lambda \simeq 1.06 \mu \mathrm{m}$, delivered energies of $200 \mathrm{~J}$ on the target in pulses of $750 \mathrm{fs}$ duration, leading to peak intensities on the target $\sim 2 \times 10^{20} \mathrm{~W} \mathrm{~cm}^{-2}$.

The ice-layered targets used in the campaign consisted of $10 \mu \mathrm{m}$ thick $\mathrm{Au}$ foils, with a $3 \pm 1 \mu \mathrm{m}$ thick layer of frozen $\mathrm{D}_{2} \mathrm{O}$ deposited on its surface. The thickness of the ice layer was characterised using 

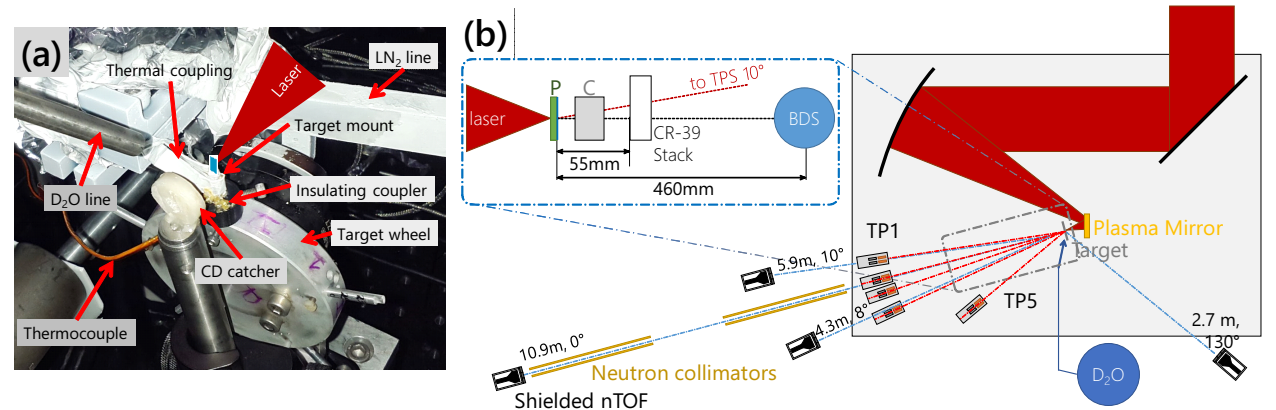

Figure 1. (a) Labelled photograph of the experimental setup inside the chamber. Heavy water vapour was released near to the rear surface of the target, which was cooled using liquid nitrogen $\left(\mathrm{LN}_{2}\right.$ line). The CD catcher disc was placed at the rear side of the target for generating neutron by the ions produced from the laser irradiated target. (b) shows a schematic of the complete experimental setup, including the suite of diagnostics employed for spectra and angular characterisation of ions and neutrons. The Thomson Parabola spectrometers, from TP1 to TP5, where distributed at angles $-6^{\circ}, 0^{\circ}, 4^{\circ}, 10^{\circ}$, and $25^{\circ}$ with respect to the laser axis. The insert shows the schematic of additional diagnostics (CR-39 and BDS) used for characterisation of the neutron beam while using the pitcher-catcher configuration.

thin-film interference reflectometry. The in-chamber setup used for the ice deposition is shown in Fig. 1(a), which, as discussed by Krygier et al. [33], is mainly based on releasing a small amount of $\mathrm{D}_{2} \mathrm{O}$ vapour directed towards the rear surface of a cryogenicallycooled $10 \mu \mathrm{m}$ thick $\mathrm{Au}$ foil. In order to compare the ion and neutron production from the ice-layered targets, several shots were taken with plain Au foils (without an ice layer) and ice-layered targets with and without a secondary catcher at the target rear side. The catcher target, consisted of a $2 \mathrm{~cm}$ thick $\mathrm{CD}$ cylinder placed at $5 \mathrm{~mm}$ behind the pitcher target, was deployed in order to enhance the neutron yield by light-ion fusion reactions inside the catcher.

The ion beams produced by the irradiation of these targets were characterised using Thomson Parabola Spectrometers (TPS), as shown in Fig. 1(b). The TPSs were used to characterise the multi-species ion spectrum along different directions. A differential filter [36] was placed on top of the TPS detector (BAS-TR image plates [37]) in order to characterise the deuterium ions, which would otherwise overlap with other ion species of same charge to mass ratio (for ex. the $\mathrm{O}^{8+}$ from the heavy ice layer).

The neutron beam was characterised by a suite of diagnostics fielded around the interaction point. Absolutely-calibrated neutron scintillators [38] were deployed in a Time-of-Flight (nToF) arrangement, at the farthest possible distance from the target (see Fig. 1(b) for positions and distances), and were shielded against Bremsstrahlung radiation produced in and around the laser-plasma interaction region, by using $10 \mathrm{~cm}$ of lead surrounding the detectors. The (distance, angle with respect to laser axis) of the four $\mathrm{nToF}$ detectors used in this paper were positioned at $\left(5.9 \mathrm{~m}, 10^{\circ}\right),\left(10.9 \mathrm{~m}, 0^{\circ}\right),\left(4.3 \mathrm{~m}, 8^{\circ}\right)$ and $(2.7 \mathrm{~m}$, $\left.130^{\circ}\right)$. The on-axis neutron spectra obtained via $\mathrm{nToF}$ were complemented by absolutely calibrated Bubble Detector Spectrometers (BDS) [39], which provide neutron fluxes in six energy bins between 0.01 and $20 \mathrm{MeV}$. The neutron beam profile was measured using CR-39 nuclear track detectors [40], placed at a short distance $(2 \mathrm{~cm})$ from the rear side of the catcher. The CR-39s were shielded using $\sim 5 \mathrm{~mm}$ lead to avoid possible exposure by the ions produced from the pitcher target. After exposure, the CR-39s were etched using a $6.5 \mathrm{M} \mathrm{NaOH}$ solution maintained at a temperature of $85^{\circ} \mathrm{C}$. Spatially-resolved neutron flux was estimated from the CR-39 data using the calibration reported by Frenje et al. [41].

\section{Characterisation of the deuterium-rich ion beam from ice-layered targets}

A typical TPS traces of ion beam obtained from the irradiation of a plain Au foil is shown in Fig. 2(a). As expected, the multi-species ion beam produced from this interaction is dominated by protons, with a minor contribution from other heavier species from the hydrocarbon and water vapour contaminants present on the rear surface of the target. The traces of the heavier ions can be seen in the gaps between the filters, as the differential filtering used in front of the detector was designed to stop ion species heavier than deuteron.

In order to accelerate deuterons via TNSA from thin foil targets, the usual practice is using deuteriumrich targets, such as deuterised plastic (CD) foils, in which $\sim 99 \%$ of the hydrogen atoms are substituted by deuterium atoms. The ion beam obtained from the irradiation of a $10 \mu \mathrm{m} \mathrm{CD} \mathrm{target} \mathrm{is} \mathrm{shown} \mathrm{in}$ Fig. 2(b), where one can see a significant flux of high energy deuterons being accelerated along with the protons. However, as it can be seen from the ion spectra shown in Fig. 2(d), the proton flux (green) is 

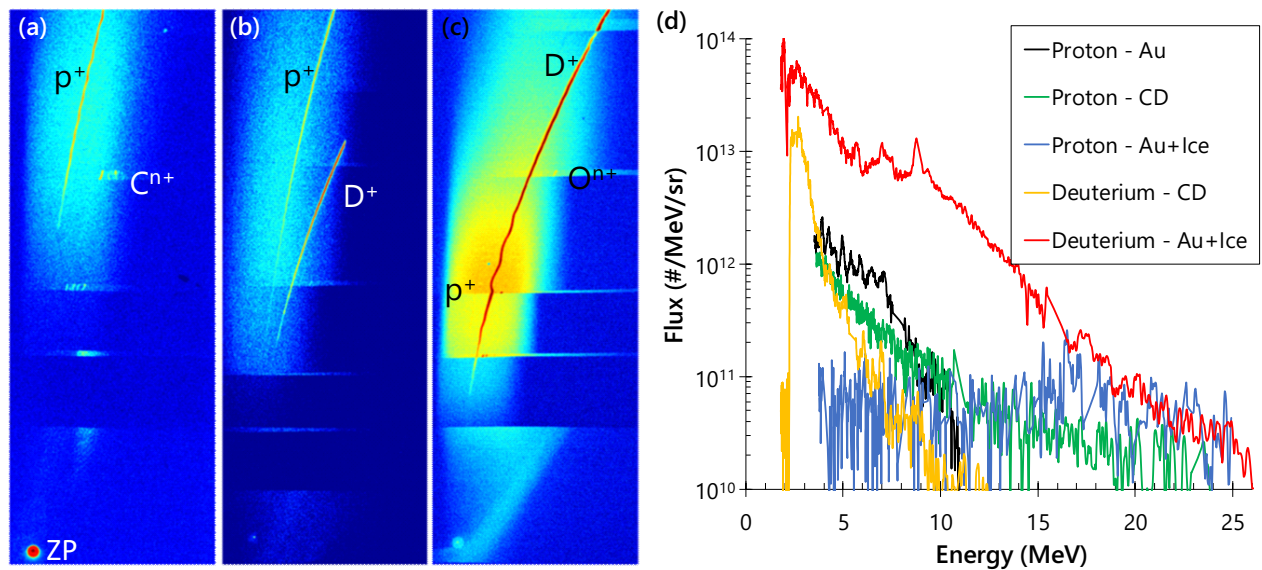

Figure 2. (a-c) Raw image plate data from the on-axis Thomson parabola spectrometer obtained from the irradiation of (a) plain $\mathrm{Au}$ foil, (b) thin CD foil, and (c) $\mathrm{D}_{2} \mathrm{O}$-covered $\mathrm{Au}$ foil. (d) Ion spectra for protons and deuterons calculated from (a-c).

similar to that obtained from the Au target (black), constituting a major fraction of the net laser-ion conversion efficiency. Although these multi-species ion beams have been employed for producing neutron beams $[25,8]$, a proton-free contamination layer at the target rear surface would lead to the acceleration of the deuterons more efficiently, which could lead optimising the neutron yield via the aforementioned $(\mathrm{d}, \mathrm{n})$ reactions $[28,24]$.

Unlike the proton-rich ion beams produced by the $\mathrm{Au}$ and $\mathrm{CD}$ targets, the ice-layered Au foil produced an almost proton-free ion beam as shown in Fig. 2(c) [33]. In our case the hydrocarbon contaminants were buried underneath a $\mathrm{D}_{2} \mathrm{O}$ ice layer, which produced ion beams highly dominated by deuterons, as shown in Fig. 2(c), with an estimated laser-to-deuteron energy conversion efficiency of $\sim 9 \%$ and a maximum deuteron energy up to $29 \mathrm{MeV}$ [33]. The absolute spectra for both proton and deuterium ions obtained from the icelayered target are shown in Fig. 2(d), which shows a significant enhancement in deuteron flux and energy compared to the CD targets, while the proton spectra was suppressed by orders of magnitude compared to both the $\mathrm{Au}$ and $\mathrm{CD}$ targets. Integrating the spectrum above the detection threshold of our TPS $\left(E_{d} \geq 1\right.$ $\mathrm{MeV} / \mathrm{n})$, deuteron flux along the target normal is estimated to be $\sim 1.6 \times 10^{14} \mathrm{~d}^{+} /$sr for the case of icelayered target, which is an order of magnitude higher than that obtained from a CD foil $\left(\sim 1.4 \times 10^{13} \mathrm{~d}^{+} / \mathrm{sr}\right)$.

\section{Neutron generation}

As mentioned in section 1, one of the motivations behind optimising the deuteron sources is neutron production via light-ion fusion reaction. In parallel with the ion beam characterisation, neutron ToF detectors were deployed to characterise neutron spectra along different angles with respect to the the laser axis (as shown in Fig 1). Furthermore, a full suite of neutron detectors, as discussed in section 2, was employed in a number of dedicated shots in order to obtain full spectral and spatial profiles of the neutron beams generated from different target configurations. The data obtained from different shots are summarised in Fig. 3.

In comparison to the case of a $\mathrm{Au}$ foil without the ice layer, one can clearly see (Fig. 3(a)) a significant increase in neutron flux and energy from a ice-layered target. The signal obtained from the reference $\mathrm{Au}$ foil accounts for the neutrons generated from proton, electron and gamma induced nuclear reactions within and surrounding the target, which is clearly insignificant (close to the detection threshold of the detectors, which for the data presented in the paper was notionally set to 2 times of the standard deviation of the noise in the signal trace) compared to those produced by the deuterated ice-layered targets. The high flux of neutrons obtained from the icelayered target can be attributed to deuterium induced reactions taking place at both front and rear sides of the target. The ice layer on the target was formed by cooling the target below freezing temperature and creating a $\mathrm{D}_{2} \mathrm{O}$ vapour ambience in proximity to the target. It is therefore conceivable that ice layers were formed on both sides of the target foil. Although a detail characterisation of the ice layer on the target front surface was not performed in the campaign, significant amount of deuterons are expected to be accelerated towards both forward (by the hole boring mechanism) and backward (by the sheath field produced by hot electrons) directions at the target front surface. In principle, these ions can contribute towards the overall neutron production by initiating fusion reactions with both the neighbouring 
(a)

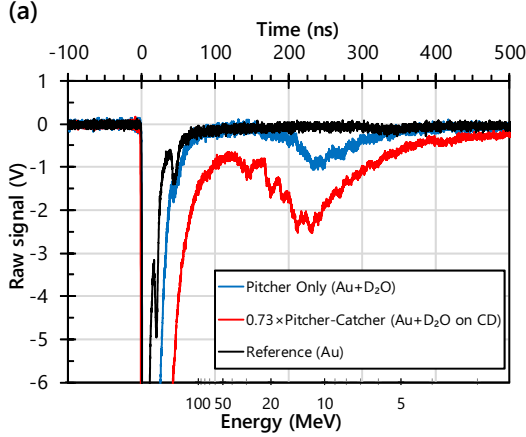

(b) Pitcher-only

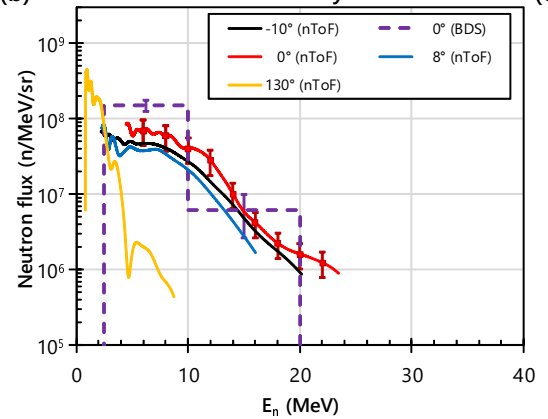

(c)

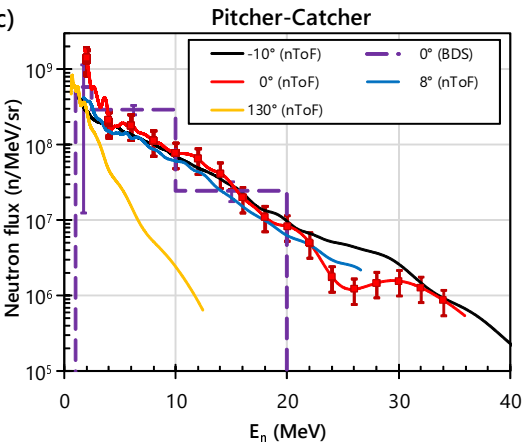

Figure 3. (a) shows the comparison between raw signals from the on-axis ToF scintillator detector, obtained from reference Au foil shot (black), a foil with $\mathrm{D}_{2} \mathrm{O}$ layer deposited (blue) and a ice-layered pitcher - CD catcher shot (red). The raw signals were normalised taking into account the difference in the gain of the detector on shots. (b) and (c) show the comparison between neutron spectra, measured along different angles with respect to the laser axis, for the ice-layered pitcher only and ice-layered pitcher-catcher shots, respectively. The neutron spectra are obtained by using a detection threshold for the signal as 2 times the standard deviation of the noise in the signal trace.

deuterons inside the plasma cloud and the ambient $\mathrm{D}_{2} \mathrm{O}$ vapour. Similar situation coexists at the target rear surface, driven by the TNSA driven deutrons discussed earlier. It is difficult to estimate at this stage the relative contribution of the neutrons generated at the front and rear of the target towards the overall neutron flux measured in the campaign. Nevertheless, it is most likely that the higher energy $(10 \mathrm{~s} \mathrm{MeV})$ neutrons of higher flux observed along the forward direction are produced by the highest energy deutrons accelerated from the target rear surface via TNSA mechanism. As shown in ref. [33], the ice-layered target typically produced deuterons upto $30 \mathrm{MeV} /$ nucleon, which, based on the reaction kinematics, is consistent with the highest energy neutrons observed along the beam axis (see Fig. 3(b)). In terms of neutron yield, the ice-layered targets produced on-axis flux of $\sim(6 \pm 2) \times 10^{8} \mathrm{n} / \mathrm{sr}$ for neutron energies above $2.5 \mathrm{MeV}$, which is significantly higher than that reported previously from shots without catcher (for instance while irradiating deuterated plastic foils under similar laser conditions [25, 8]). The degree of anisotropy in neutron emission (the ratio between on axis and $130^{\circ}$ off axis neutron flux), which increases with increase in projectile ion energies [8], also suggests that the neutron generation from the ice-layered target is mainly due to the fusion reactions triggered by the high energy deuterons from the target rear.

A further increase (by a factor of 3 ) in neutron flux was achieved by efficiently converting the high energy ions into neutrons using a secondary catcher target. The increase in neutron signal in presence of a solid CD catcher can be seen in Fig. 3(a). A highly beamed (anisotropy ratio $\sim 9$ ) neutron flux $(1.87 \pm 0.7) \times 10^{9} \mathrm{n} / \mathrm{sr}$ for neutron energies above $2.5 \mathrm{MeV}$ was obtained along the forward direction, which is amongst the highest neutron fluxes reported in literature[7, 8]. Specifically, this represent nearly a three fold enhancement in neutron flux with respect to the experiment reported in ref. [8] using $\mathrm{CD}$ pitcher and CD catcher at similar laser irradiance on the same laser facility.

While the $\mathrm{d}(\mathrm{d}, \mathrm{n})^{3} \mathrm{He}$ reaction in a beam-fusion scenario mainly produces neutrons above $2.5 \mathrm{MeV}$, the lower energy neutrons can be produced by other deuterium-induced reactions, such as deuterium breakup, ${ }^{12} \mathrm{C}(\mathrm{d}, \mathrm{n}){ }^{13} \mathrm{~N}[1,42,28]$. As shown in Fig. 3(c), neutrons of energy upto $\sim 40 \mathrm{MeV}$ were measured along the beam forward direction, also higher (by a factor of $\sim 2$ ) than the energies obtained while using $\mathrm{CD}$ foils as pitcher in ref. [8]. According to the kinematic of the $\mathrm{d}(\mathrm{d}, \mathrm{n})^{3} \mathrm{He}$ reaction $\left(E_{n}=\right.$ $\left(E_{d} / 8\right)\left(1+\sqrt{3+3 Q / E_{d}}\right)^{2}[6,8,43]$, where $E_{d}$ and $E_{n}$ represent the deuterium and neutron energies respectively along the axis and $Q$ the $\mathrm{Q}$-value of the reaction), such neutron energy would be expected from a beam-fusion scenario employing $\sim 36 \mathrm{MeV}$ deuterons, which is similar to the maximum deuterium energy measured experimentally from the ice-layered targets [33].

In order to characterise in detail the angular emission profile of the neutrons, and to complement the anisotropy measurements by the ToF detectors, a CR39 stack was deployed in close proximity to the catcher. The neutron beam profile shown in Fig. 4 was obtained by counting the number of neutron-induced pits and using the calibration reported by Frenje et al. [41]. As can be seen, the neutron flux profile obtained from the CR39 is in good agreement with the data obtained from BDS and ToF dectectors. The difference between on- and off-axis neutron fluxes is clearly noticeable in the raw images of the CR39 surface, as shown in the inserts. The FWHM divergence of the neutron beam can be measured as $\sim 70^{\circ}$, which is similar to the 


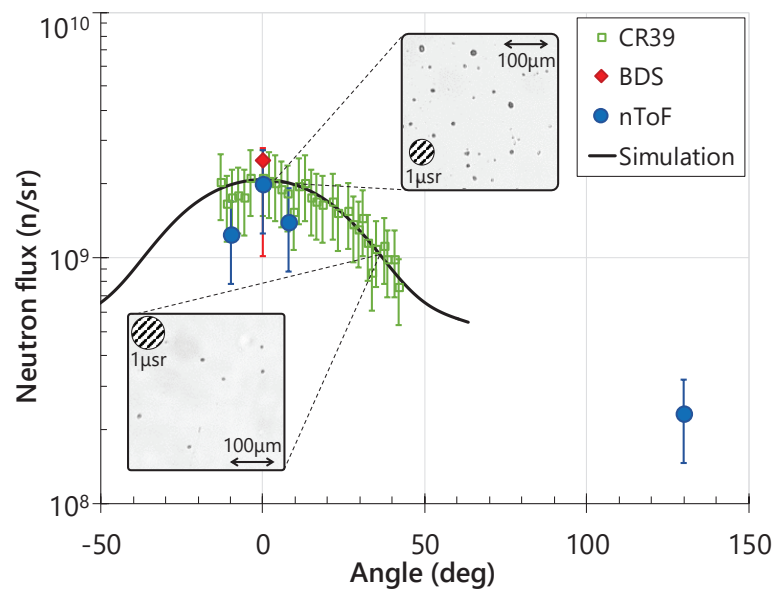

Figure 4. Neutron beam profile experimentally measured from a line-out on a CR-39 detector (green), compared with simultaneous measurements using nToF scintillators (blue) and BDS (red). Insets show microscope views of the neutron pits on the CR-39 surface at two different positions. The experimental measurements are compared with a numerical simulation of the neutron generation from the characterised laser-driven deuterium ion beam (black).

beam divergences obtained by Kar et al. [8] employing TNSA-driven ions (protons and deuterons) in a beamfusion scenario.

In order to substantiate the neutron beam profile obtained experimentally, the beam-fusion scenario was simulated using the model discussed by Alejo et al. in ref. [34]. The modelling was facilitated by the angularly resolved characterisation of the deuteron beam spectral profiles from a ice-layered target, and based on the premise that the neutrons above $2.5 \mathrm{MeV}$ are mainly produced by the $\mathrm{d}(\mathrm{d}, \mathrm{n})^{3} \mathrm{He}$ reaction due to the lack of protons in the accelerated ion beam from the ice-layered target. The input beam of deuteron in the simulation was modelled by interpolating the spectra obtained by the TPSs deployed along different angles, and by assuming a smooth spatial profile typical to the TNSA-driven beams. As shown in Fig. 4, the simulation reproduces the experimental data which underpins the underlying neutron generation mechanism. Such beamed emission of neutrons with high peak flux is greatly useful for its direct applications as well as for further transport and moderation. Nevertheless, there is significant scope for optimising the peak flux by deploying higher yield catcher materials (for ex. Lithium) [9, 24].

\section{Conclusions}

Laser driven neutron sources are currently attracting a great deal of interest, pursued by many groups across the globe, constantly trying to find ways to optimize the neutron beam parameters. A highly-directional and bright neutron source was demonstrated by employing nearly-pure deuterium source from $\mathrm{D}_{2} \mathrm{O}$ coated thin targets irradiated by petawatt laser pulses. High energy and high flux deuteron and neutron beams were characterised both spatially and spectrally by deploying a full suite of ion and neutron diagnostics. Peak fluxes on the order of $10^{9} \mathrm{n} / \mathrm{sr}$ for neutrons of energy above $2.5 \mathrm{MeV}$, with a divergence cone of $\sim$ $70^{\circ}$ FWHM, was obtained while using a CD catcher behind the ice-layered target. The neutron beam profile obtained in the experiment was reproduced by numerical simulations while using experimentally measured deuteron spectra.

Unlike the typical CD targets, which produces ion beams dominated by protons (being the species with highest charge-to mass ratio, protons are preferentially accelerated by the TNSA mechanism), the $\mathrm{D}_{2} 0$ layered targets produce significantly higher flux of higher energy deuterium ions. Such enriched source of pure deuterium can, in principle, lead to a significant increase in neutron flux by using higher yield neutron converters, such as Li and Be.

\section{Acknowledgements}

Authors would like to acknowledge funding from EPSRC, through Grants EP/J002550/1-Career Acceleration Fellowship held by S.K., EP/L002221/1, and EP/K022415/1. A.G.K. and R.R.F. acknowledge funding from the United States Department of Energy through Contract No. DE-FC02-04ER54789. J.T.M. acknowledges funding from the Quantum and NonEquilibrium Processes Department of the Air Force Office of Scientific Research, under the management of Dr. Enrique Parra, Program Manager. Support from laser operators, engineers, and technicians at Rutherford Appleton Laboratory is also acknowledged.

\section{References}

[1] Norreys P, Fews A, Beg F, Bell A, Dangor A, Lee P, Nelson M, Schmidt H, Tatarakis M and Cable M 1998 Plasma physics and controlled fusion 40 175-182

[2] Disdier L, Garconnet J, Malka G and Miquel J 1999 Physical review letters $\mathbf{8 2} 1454$

[3] Fritzler S, Najmudin Z, Malka V, Krushelnick K, Marle C, Walton B, Wei M, Clarke R and Dangor A 2002 Physical review letters 89165004

[4] Lancaster K L, Karsch S, Habara H, Beg F N, Clark E L, Freeman R, Key M H, King J A, Kodama R, Krushelnick $\mathrm{K}$, Ledingham $\mathrm{K} \mathrm{W} \mathrm{D}$, McKenna $\mathrm{P}$, Murphy C D, Norreys P A, Stephens R, Steckl C, Toyama Y, Wei M S and Zepf M 2004 Physics of Plasmas 11 3404-3408

[5] Willingale L, Petrov G, Maksimchuk A, Davis J, Freeman R, Joglekar A, Matsuoka T, Murphy C, Ovchinnikov V, Thomas A et al. 2011 Physics of Plasmas 18083106

[6] Davis J and Petrov G 2008 Plasma Physics and Controlled Fusion 50065016

[7] Roth M, Jung D, Falk K, Guler N, Deppert O, Devlin M, Favalli A, Fernandez J, Gautier D, Geissel M, Haight R, 
High flux, beamed neutron sources employing deuteron-rich ion beams from $\mathrm{D}_{2} \mathrm{O}$-ice layered targets

Hamilton C E, Hegelich B M, Johnson R P, Merrill F, Schaumann G, Schoenberg K, Schollmeier M, Shimada T, Taddeucci T, Tybo J L, Wagner F, Wender S A, Wilde C H and Wurden G A 2013 Phys. Rev. Lett. 110(4) 044802

[8] Kar S, Green A, Ahmed H, Alejo A, Robinson A, Cerchez M, Clarke R, Doria D, Dorkings S, Fernandez J et al. 2016 New Journal of Physics 18053002

[9] Higginson D P, McNaney J M, Swift D C, Petrov G M, Davis J, Frenje J A, Jarrott L C, Kodama R, Lancaster K L, Mackinnon A J, Nakamura H, Patel P K, Tynan G and Beg F N 2011 Physics of Plasmas 18100703

[10] Petrov G, Higginson D, Davis J, Petrova T B, McNaney J, McGuffey C, Qiao B and Beg F 2012 Physics of Plasmas 19093106

[11] Macchi A, Borghesi M and Passoni M 2013 Reviews of Modern Physics 85751

[12] Esirkepov T, Borghesi M, Bulanov S, Mourou G and Tajima T 2004 Physical Review Letters 92175003

[13] Kar S, Kakolee K, Qiao B, Macchi A, Cerchez M, Doria D, Geissler M, McKenna P, Neely D, Osterholz J et al. 2012 Physical Review Letters 109185006

[14] Yin L, Albright B, Hegelich B and Fernández J 2006 Laser and Particle Beams 24 291-298

[15] Yin L, Albright B, Hegelich B, Bowers K J, Flippo K, Kwan $\mathrm{T}$ and Fernández J 2007 Physics of plasmas 14056706

[16] Powel H, King M, Gray R, MacLellan D, GonzalezIzquierdo B, Stockhausen L, Hicks G, Dover N, Rusby D, Carroll D, Padda H, Torres R, S K, Clarke R J, Musgrave I, Najmudin Z, Borghesi M, Neely D and McKenna P 2015 New J. Physics 17103033

[17] Dover N P, Palmer C A J, Streeter M J V, Ahmed H, Albertazzi B, Borghesi M, Carroll D C, Fuchs J, Heathcote R, Hilz P, Kakolee K F, Kar S, Kodama R, Kon A, MacLellan D A, McKenna P, Nagel S R, Neely D, Notley M M, Nakatsutsumi M, Prasad R, Scott G, Tampo M, Zepf M, Schreiber J and Najmudin Z 2016 New Journal of Physics $\mathbf{1 8} 013038$

[18] Kar S, Ahmed H, Prasad R, Cerchez M, Brauckmann S, Aurand B, Cantono G, Hadjisolomou P, Lewis C L, Macchi A et al. 2016 Nature communications 710792

[19] Kar S, Ahmed H, Nersisyan G, Brauckmann S, Hanton F, Giesecke A L, Naughton K, Willi O, Lewis C L S and Borghesi M 2016 Physics of Plasmas 23055711

[20] Gitomer S, Jones R, Begay F, Ehler A, Kephart J and Kristal R 1986 Physics of Fluids 29 2679-2688

[21] Wilks S, Langdon A, Cowan T, Roth M, Singh M, Hatchett S, Key M, Pennington D, MacKinnon A and Snavely R 2001 Physics of Plasmas 8 542-549

[22] Higginson D P, Vassura L, Gugiu M M, Antici P, Borghesi M, Brauckmann S, Diouf C, Green A, Palumbo L, Petrascu H, Sofia S, Stardubtsev M, Willi O, Kar S, Negoita F and Fuchs J 2015 Phys. Rev. Lett. 115(5) 054802

[23] Brenner C M, Mirfayzi S R, Rusby D R, Armstrong C, Alejo A, Wilson L A, Clarke R, Ahmed H, Butler N M H, Haddock D, Higginson A, McClymont A, Murphy C, Notley M, Oliver P, Allott R, Hernandez-Gomez C, Kar S, McKenna P and Neely D 2016 Plasma Physics and Controlled Fusion $\mathbf{5 8} 014039$

[24] Davis J, Petrov G, Petrova T, Willingale L, Maksimchuk A and Krushelnick K 2010 Plasma Physics and Controlled Fusion $\mathbf{5 2} 045015$

[25] Alejo A, Ahmed H, Green A, Mirfayzi S, Borghesi M and Kar S 2016 Nuovo Cimento C $\mathbf{3 8} 188$

[26] Izumi N, Sentoku Y, Habara H, Takahashi K, Ohtani $\mathrm{F}$, Sonomoto T, Kodama R, Norimatsu T, Fujita H, Kitagawa Y et al. 2002 Physical Review E 65036413

[27] Hou B, Nees J A, He Z, Petrov G, Davis J, Easter J H, Thomas A G and Krushelnick K M 2011 Physics of

\section{Plasmas 18040702}

[28] Maksimchuk A, Raymond A, Yu F, Petrov G, Dollar F, Willingale L, Zulick C, Davis J and Krushelnick K 2013 Applied Physics Letters 102191117

[29] Morrison J, Storm M, Chowdhury E, Akli K, Feldman S, Willis C, Daskalova R, Growden T, Berger P, Ditmire T et al. 2012 Physics of Plasmas 19030707

[30] Hegelich M, Karsch S, Pretzler G, Habs D, Witte K, Guenther W, Allen M, Blazevic A, Fuchs J, Gauthier $\mathrm{J}$ et al. 2002 Physical review letters $\mathbf{8 9} 085002$

[31] McKenna P, Lindau F, Lundh O, Carroll D C, Clarke R J, Ledingham K, McCanny T, Neely D, Robinson A P L, Robson L, Simpson P T, Wahlstrom C G, Zepf M and Hegelich B 2007 Plasma Physics Controlled Fusion 49 B223

[32] Allen M, Patel P K, Mackinnon A, Price D, Wilks S and Morse E 2004 Phys. Rev. Lett. 93(26) 265004

[33] Krygier A, Morrison J, Kar S, Ahmed H, Alejo A, Clarke R, Fuchs J, Green A, Jung D, Kleinschmidt A et al. 2015 Physics of Plasmas 22053102

[34] Alejo A, Green A, Ahmed H, Robinson A, Cerchez M, Clarke R, Doria D, Dorkings S, Fernandez J, McKenna $\mathrm{P}$ et al. 2016 Nuclear Instruments and Methods in Physics Research Section A: Accelerators, Spectrometers, Detectors and Associated Equipment 829 176

[35] Danson C, Brummitt P, Clarke R, Collier J, Fell B, Frackiewicz A, Hancock S, Hawkes S, Hernandez-Gomez C, Holligan P, Hutchinson M, Kidd A, Lester W, Musgrave I, Neely D, Neville D, Norreys P, Pepler D, Reason C, Shaikh W, Winstone T, Wyatt R and Wyborn B 2004 Nuclear Fusion 44 S239

[36] Alejo A, Kar S, Ahmed H, Krygier A, Doria D, Clarke R, Fernandez J, Freeman R, Fuchs J, Green A et al. 2014 Review of Scientific Instruments $\mathbf{8 5} 093303$

[37] Fujifilm bas-tr imaging plates http://www.fujifilm.com

[38] Mirfayzi S, Kar S, Ahmed H, Krygier A, Green A, Alejo A, Clarke R, Freeman R, Fuchs J, Jung D et al. 2015 Review of Scientific Instruments $\mathbf{8 6} 073308$

[39] Bubble technology industries inc. http://bubbletech.ca/

[40] Fleischer R, Price P and Walker R 1965 Journal of applied Physics 36 3645-3652

[41] Frenje J A, Li C K, Séguin F H, Hicks D G, Kurebayashi S, Petrasso R D, Roberts S, Glebov V Y, Meyerhofer D D, Sangster T C, Soures J M, Stoeckl C, Chiritescu C, Schmid G J and Lerche R A 2002 Review of scientific instruments 73 2597-2605

[42] Youssef A and Kodama R 2010 Nuclear Fusion 50035010

[43] Toupin C, Lefebvre E and Bonnaud G 2001 Physics of Plasmas 8 1011-1021 